\title{
The other-race and other-species effects in face perception - a subordinate-level analysis
}

\author{
Christoph D. Dahl' ${ }^{1}$, Malte J. Rasch ${ }^{2,3}$ and Chien-Chung Chen ${ }^{1}$ \\ ' Department of Psychology, National Taiwan University, Taipei, China \\ 2 State Key Laboratory of Cognitive Neuroscience and Learning \& IDG/McGovern Institute for Brain Research, Beijing Normal University, Beijing, China \\ ${ }^{3}$ Center for Collaboration and Innovation in Brain and Learning Sciences, Beijing Normal University, Beijing, China
}

\section{Edited by:}

Snehlata Jaswal, Indian Institute of

Technology, Jodhpur, India

Reviewed by:

Maria Olkkonen, University of

Pennsylvania, USA

Sarina Hui-Lin Chien, Graduate

Institute of Neural and Cognitive

Sciences - China Medical University,

Taiwan

\section{*Correspondence:}

Christoph D. Dahl, Department of Psychology, National Taiwan

University, Taipei 10617, Taiwan, China e-mail:christoph.d.dahl@gmail.com
The ability of face discrimination is modulated by the frequency of exposure to a category of faces. In other words, lower discrimination performance was measured for infrequently encountered faces as opposed to frequently encountered ones. This phenomenon has been described in the literature: the own-race advantage, a benefit in processing own-race as opposed to the other-race faces, and the own-species advantage, a benefit in processing the conspecific type of faces as opposed to the heterospecific type. So far, the exact parameters that drive either of these two effects are not fully understood. In the following we present a full assessment of data in human participants describing the discrimination performances across two races (Asian and Caucasian) as well as a range of non-human primate faces (chimpanzee, Rhesus macaque and marmoset). We measured reaction times of Asian participants performing a delayed matching-to-sample task, and correlated the results with similarity estimates of facial configuration and face parts. We found faster discrimination of own-race above other-race/species faces. Further, we found a strong reliance on configural information in upright own-species/-race faces and on individual face parts in all inverted face classes, supporting the assumption of specialized processing for the face class of most frequent exposure.

Keywords: face perception, other-race effect, other-species effect, own-race advantage, own-species advantage, similarity, configural processing, heterospecific faces

\section{INTRODUCTION}

Humans as well as other primates are able to extract information from faces to infer invariant properties such as race (Michel etal., 2007; Tanaka and Pierce, 2009), species (Pascalis et al., 2002; Dufour et al., 2006), gender (Bulthoff, 2009), age (Kwon and da Vitoria Lobo, 1999), nationality (Li et al., 2004), rank (Dahl and Adachi, 2013), and to some extent personality traits (Ekman etal., 1980). The most important ability is to recognize and identify faces (Marr and Nishihara, 1978; Bruce and Young, 1986). Identity is an invariant face property that, however, is not fully independent from other invariant properties, such as race and species. Humans (Meissner et al., 2005; Hugenberg etal., 2010) and other primates, such as chimpanzees (Dahl etal., 2013a) and monkeys (Dahl etal., 2007, $2009,2010,2011)$ are experts in individuating the faces of their own species and race, however, their proficiency deteriorates for faces rarely exposed to. This phenomenon has been demonstrated by superior discrimination ability for the own-race as opposed to other-race faces (OREs), known as the other-race effect (ORE), the own-race advantage or the other-race bias (Lindsay et al., 1991; Meissner and Brigham, 2001). Similarly, there is an effect that it is easier to discriminate faces of the own species than those of other species. Such effect has been referred to as own-species advantage, the other- or own-species effect (OSE; Scott and Fava, 2013). The ORE has been addressed to a great extent (Meissner and Brigham, 2001) and elicited a robust effect in observers of different races (Bothwell et al., 1989). The OSE has been studied extensively in humans, using various behavioral (Dufour etal., 2004; Dufour and Petit, 2010) and neural methodological approaches (de Haan etal., 2002; Scott etal., 2005). Humans as well as monkeys show an advantage for ownspecies faces: they were more skilled in discriminating (Pascalis and Bachevalier, 1998) and recognizing (Dufour et al., 2004) the own-species as opposed to the other-species face (OSE) class. Human adults show better detection of configural differences in facial features of human but not monkey faces. Only inverted human but not monkey faces disrupt this sensitivity (Mondloch et al., 2006).

Still, it is not clear whether the two effects can be described by the same underlying computational mechanism of the face recognition system when it is exposed to a face class other than its own. In detail, the ORE occurs at the level of two face categories that are slightly different from each other in terms of facial components and configurations (shared human morphology), while the OSE occurs at the level of two face categories that fundamentally vary in the morphological structure. This raises an interesting question: do we recruit the same processing mechanism for other-race as for own-race faces and for OSEs as for own-species faces? Does the morphological (dis)similarity influence the configural processing of these face types? We examine the ORE and the OSE using the same paradigm embedded in the same experimental procedure. In a delayed matching-to-sample task, human participants 
discriminated pairs of faces of Asian and Caucasian races as well as chimpanzee, rhesus macaque and marmoset species. We hypothesize that any morphological deviation from the default class of faces (here Asian faces) causes a disruption of discrimination performance relative to the default class. We further estimated the contribution of configural as opposed to part-based contributions by correlating discrimination performances with the similarity values drawn from pairwise comparisons of the stimuli's configural arrangement (configuration) and facial parts (part-based). Hence, we further hypothesize that the processing of own-class faces involves a relatively higher contribution of configural information as opposed to other face classes, while with increasing morphological distance from the conspecific face class, the contribution of part-based information, as in object-like processing, should increase. Further, assuming that face inversion impairs configural processing (Freire et al., 2000), we predict that inverted face comparisons of all classes show comparable performances that go along with an equal contribution of part-based information across all face classes.

\section{MATERIALS AND METHODS PARTICIPANTS}

Twenty-four human participants (10 females; 22.5 years $+/-1.5$ SD) took part in this study. Twenty-two participants participated in the task using upright and 22 using inverted stimuli. In other words, two participants did not participate in both experiments. The participants were selected from a pool of students at the National Taiwan University and consist of Asian members exclusively. The participants have never encountered the stimuli used and they have never been tested on a comparable face discrimination task. The participants were naïve to the purpose of the experiment. Written consent was obtained from each participant prior to the experiment.

\section{APPARATUS AND PROCEDURE}

We used a delayed-matching-to-sample paradigm (DMS), presenting one face stimulus centrally on the display for $300 \mathrm{~ms}$ (cue), followed by an inter-stimulus interval (ISI) of $250 \mathrm{~ms}$, followed by a mask for $300 \mathrm{~ms}$, followed by a second ISI of $500 \mathrm{~ms}$ and two face stimuli, with one showing the same picture as in the cue presentation (match) and one showing a different individual's face picture (distractor). The match and distractor were shown for $3500 \mathrm{~ms}$, however, responses could be given after disappearance of the stimuli on the display. The task was to indicate which of the two stimuli (match and distractor) is identical to the cue stimulus. In the inversion experiment, all faces, including the cue, were rotated in image-plane for $180^{\circ}$. The vertical spacing of the match and distractor stimuli was about $40 \mathrm{~mm}\left(2.92^{\circ}\right.$ of gaze angle). Stimuli were presented at a 19 -inch CRT display $(1024 \times 768$ pixels $)$ controlled by custom-written software under MATLAB (Mathworks Inc., Natick, MA, USA) and Psychtoolbox (Brainard, 1997; Pelli, 1997). Testing of upright and inverted stimuli was done block-wise and separated by maximally 3 days. Participants received financial compensation. Participants sat in an experimental room $(2.5 \mathrm{~m}$ wide, $2.5 \mathrm{~m}$ deep), facing the computer monitor at a viewing distance of $100 \mathrm{~cm}$. The viewing distance was controlled using a chin rest.

\section{STIMULI}

We used black-and-white pictures of faces of Asian and Caucasian humans as well as chimpanzees (Pan troglodytes), Rhesus macaques (Macaca mulatta) and Marmoset monkeys (Callithrix jacchus). All faces were of unfamiliar individuals. All stimuli were normalized for luminance and contrast and presented on the screen in an image canvas of 5.75 by $5.75^{\circ}$ of gaze angle. Masks were created by extracting 50 rectangular patches from the original face stimuli. The size of these patches was $13.33 \%$ of the original image dimensions. Patches were randomly placed onto a white blank surface (a canvas of the same size as the face stimuli). This procedure resulted in certain overlap of individual patches, while fully covering the blank surface. For each face category, mask stimuli were created and solely used for trials of the same type of faces. In total, we used five sets of 12 face stimuli each, resulting in 660 comparisons. In each trial, the mask was randomly chosen from a set of 50 masks. Each participant did five runs of 132 trials each consisting of all five categories intermixed and fully counter-balanced across categories and face identities.

\section{DATA ANALYSIS}

The analyses were performed using Matlab (Mathworks Inc., Natick, MA, USA). The dependent variable was reaction times and error rates. Trials with reaction times above $4000 \mathrm{~ms}$ were excluded. Only correct trials went into the analyses of reaction times, which is 547 (+/-8.26 SD) upright and 553 (+/-10.7 SD) inverted trials. The statistics follow a within-subject design. Analyses of variances were performed using a fixed effect ANOVA with stimulus category and stimulus presentation (upright, inverted) as fixed factors, an interactive analysis of covariance (ANCOVA) and two-sample $t$-tests. The similarity scores of the facial configuration within face classes were determined by calculating the mean of the Euclidean distances between corresponding configural marker points of each pair of faces. These markers were placed manually by the authors. Euclidean distance were further visualized by classical multidimensional scaling (cMDS), which aims at placing each face in $\mathrm{N}$-dimensional space such that the betweenface distances are preserved as well as possible. The maximum error is 2.68 in a two-dimensional and 2.66 in a full reconstruction. To determine the similarity between individual facial parts (eyes, nose, mouth), we used topological methods (Gunduz and Krim, 2003) that viewed each two-dimensional images as a surface with the pixel intensities represented as values on the $z$-axis. Using the second derivatives the principle curvatures of the surface were extracted. The facial features, such as eyes, nose, and mouth, were composed of valleys and crests. Areas of interests were determined by cutting the image in the horizontal dimension. The cut-off lines varied across face classes given the differential face morphology. The extracted curvature profiles were average in the horizontal dimension for each image, resulting in a feature vector. The feature vectors of individual parts were normalized across all classes to an equivalent length, i.e., number of samples. Similarity scores of facial parts were correlated with the reaction times for upright and inverted face trials separately and similarity scores of the facial configuration were correlated with the performance difference of upright and inverted face trials by subtracting the upright face trials from the inverted ones. 


\section{RESULTS}

We tested the reaction times of Asian participants on the discrimination of Asian and Caucasian human faces, as well as chimpanzee ( $P$. troglodytes), macaques ( $M$. mulatta) and marmoset monkey (C. jacchus) faces in upright and inverted orientations (Figure 1A). A cue stimulus (e.g., face 1) was centrally presented followed by a match-distractor stimulus pair (e.g., face 1 and face 2 ) of the same species (Figure 1B). We collected an average of 660 trials for upright as well as inverted presentations in 22 participants. Using a fixed effect ANOVA with stimulus class and face orientation (upright, inverted) as fixed factors and reaction times as dependent variable, we found a significant interaction of the factors stimulus class and the face orientation $[F(4,210)=2.52$, $p<0.05$, mean square error $=29388$; Figures 1 C-E $]$. In more detail, we found a systematic facilitation of discrimination for upright own-race faces (Asians) as opposed to upright OREs [Caucasians; $t(42)=-2.22, p<0.05$; mean Asians $=660.7 \mathrm{~ms}$, mean Caucasians $=726.2 \mathrm{~ms}$; one-tailed]. Similarly, we found an advantage for the same upright Asian faces (own-species) as opposed to upright other-species' faces, such as chimpanzee, macaques and marmosets $[t(86)=-4.16, p<0.001$; mean Asians $=660.7 \mathrm{~ms}$, mean chimpanzee $=748.9 \mathrm{~ms}$, mean macaque $=767.5 \mathrm{~ms}$, mean marmoset $=784.5 \mathrm{~ms}$; one-tailed, corrected for multiple comparisons]. These two effects, the own-race advantage and the own-species advantage, do not appear when the face stimuli were presented inverted $\left(180^{\circ}\right.$ image plane rotation): Asian faces (ownrace, own-species) are processed at the same speed as Caucasian faces [other-race; $t(42)=0.05, p=0.96$; mean Asians $=720.7 \mathrm{~ms}$, mean Caucasians $=718.7 \mathrm{~ms}]$ as well as OSEs $[t(86)=0.25$, $p=0.81$; mean Asians $=720.7 \mathrm{~ms}$, mean chimpanzee $=712 \mathrm{~ms}$, mean macaque $=717.9 \mathrm{~ms}$, mean marmoset $=710 \mathrm{~ms}]$. At the same time, inversion caused a significant increase of reaction times for Asian faces [own-race, own-species; $t(42)=-1.74$, $p<0.05$; mean upright Asians $=660.7 \mathrm{~ms}$, mean inverted Asians $=720.7 \mathrm{~ms}$; one-tailed], but no change in reaction times for Caucasian [other-race; $t(42)=0.22, p=0.83$; mean upright Caucasians $=726.2 \mathrm{~ms}$, mean inverted Caucasians $=718.7 \mathrm{~ms}]$, chimpanzee [other species; $t(42)=1.05, p=0.30$; mean upright chimpanzee $=748.9 \mathrm{~ms}$, mean inverted chimpanzee $=712 \mathrm{~ms}]$ and macaque faces [other species; $t(42)=1.46, p=0.15$; mean upright macaque $=767.5 \mathrm{~ms}$, mean inverted macaque $=717.8 \mathrm{~ms}]$. Inverted presentations of marmoset faces (other species), however, caused a facilitation in comparison to the upright presentations of the same faces $[t(42)=2.12, p<0.05$; mean upright marmoset $=784.5 \mathrm{~ms}$, mean inverted marmoset $=710 \mathrm{~ms}]$.

We further examined relative changes of reaction times by normalizing the data samples for each face class by dividing them with the grand mean of all face classes. We found that the scores decreased with evolutionary distance from Asian faces: (1) scores for Asian faces were greater than those for Caucasian faces, reflecting the ORE $[t(42)=30.70, p<0.001$; mean scores Asian $=0.11$; mean scores Caucasian $=0.02]$; (2) scores for Caucasian faces were greater than those for chimpanzee faces $[t(42)=9.64, p<0.001$; mean scores Caucasian $=0.02$; mean scores chimpanzee $=-0.02]$; (3) scores for chimpanzee faces were greater than those for macaque faces $[t(42)=4.62$, $p<0.001$; mean scores chimpanzee $=-0.02$; mean scores macaque $=-0.04]$; and (4) scores for macaque faces were greater than those for marmoset faces $[t(42)=14.34, p<0.001$; mean scores macaque $=-0.04$; mean scores marmoset $=-0.07]$. This trend is consistent across all participants (Figure 1F).

Using an interactive ANCOVA with stimulus class and face orientation (upright, inverted) as fixed factors, error rates as covariate, and reaction times as dependent variable, we found no significant effects of error rates on stimulus class and face orientation (all $p$-values $>0.26$ ). Hence, we rule out a speedaccuracy trade-off. For further analyses we focused on reaction times.

To determine whether there is a relation between the similarity of a stimulus pair and the discrimination performances we calculated similarity values for each face stimulus based on (1) the facial configuration and (2) individual parts (eyes, nose, and mouth). (1) We determined configural similarities among individual faces of the same face classes by calculating the Euclidean distances between corresponding marker-points set in the 2D face plane (Figure 2A). These values reflect the overall structure of the face, but do not include any image information. We determined similarity values between individual face configurations. These values were then correlated with the corresponding reaction times for each face class separately. For each participant reaction times were normalized by the grand mean and assigned to 20 bins of equal sizes ranging from the minimal to the maximal sample value. We analyzed upright and inverted faces separately. A negative correlation between reaction times and similarity of facial configuration would indicate that to discriminate two faces is more difficult the more similar two face configurations are. We found a negative correlation between the normalized reaction times of upright faces and the similarity scores in Asian $(r=-0.65, p<0.01)$ and Caucasian faces $(r=-0.49, p<0.05)$, but not in any OSE class (chimpanzee $r=-0.04, p=0.85$; macaque $r=0.35, p=0.18$; marmoset $r=-0.34, p=0.16$; Figure 2B). We did not find any negatively correlated relationship between the normalized reaction times of inverted faces and the similarity scores in any of the face classes (all $p>0.36$; Figure 2B). (2) We further extracted face features using methods suggested in computer vision (Gunduz and Krim, 2003). This approach treats the face image as a surface with eyes, nose and mouth being singularities in the surface that build valleys and ridges of the luminance landscapes (Figure 3). Using so-called ravines facial features can be extracted from the surface. We extracted eyes, nose and mouth regions and compared the vector profiles of these features among the faces of each face class. We determined the Euclidean distances between corresponding points of the two vector profiles being compared and correlated these similarity scores with the normalized reaction times of upright and inverted face discrimination trials (Figures 4A,B). Importantly, in contrast to the configural similarity, the part similarity was determined by actual image content, i.e., vector profiles were extracted from the image surface. The assumption is that a negative correlation between reaction times and similarity values for a certain face part would occur, if participants strongly rely on that face part and, thus, are negatively affected if two faces have closely similar parts. We found negative correlations between the eyes and the similarity scores for all classes when the faces were presented upright (Asian: $r=-0.69, p=0.01$; Caucasian: $r=-0.55$, 
A

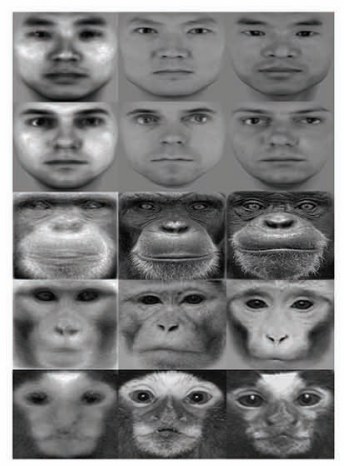

Asian

Caucasian

Chimpanzee

Macaque

Marmoset

C

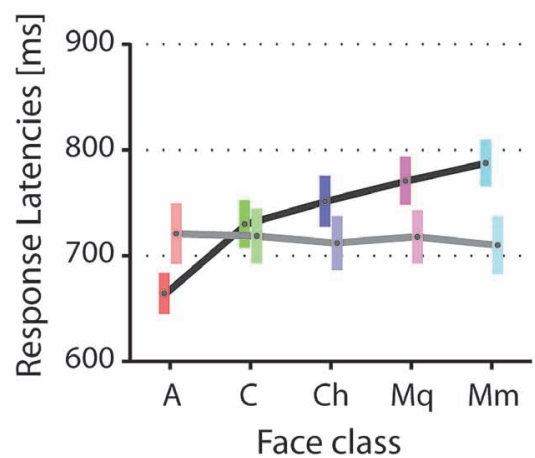

E

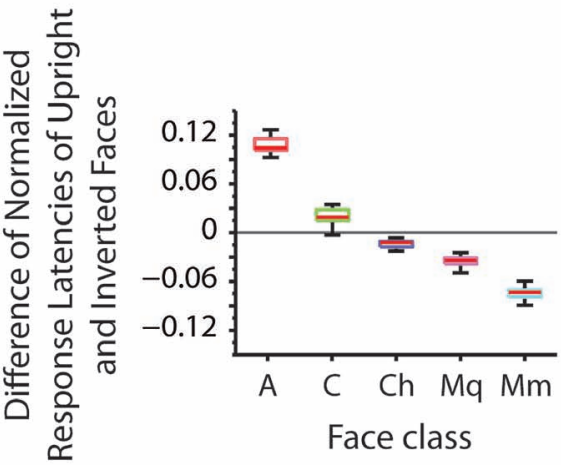

FIGURE 1 | Stimuli, procedure and reaction times, and configural analysis. (A) Stimuli: identities (horizontal) and classes (vertical). (B) Procedure. In each trial, a face picture of an individual (cue) was centrally presented on the display for $300 \mathrm{~ms}$, followed by an inter stimulus interval (ISI) of $250 \mathrm{~ms}$, followed by a mask for $300 \mathrm{~ms}$, followed by a second ISI of $500 \mathrm{~ms}$ and a presentation of two horizontally aligned face pictures of the same individual (match) and a different individual (distractor). Participants indicated their choice by pressing one of two buttons on the joystick, corresponding to the left and right stimulus on the screen. The correct answer (match) is the identical face picture as shown in the cue stimulus. (C)

Absolute reaction times were averaged across participants for each face class (A, Asian; C, Caucasian; Ch, chimpanzee; Mq, macaque; Mm, marmoset). The color bars indicate the range within the standard errors; the gray dots indicate the means. Reaction times for upright faces are shown in darker colors; those
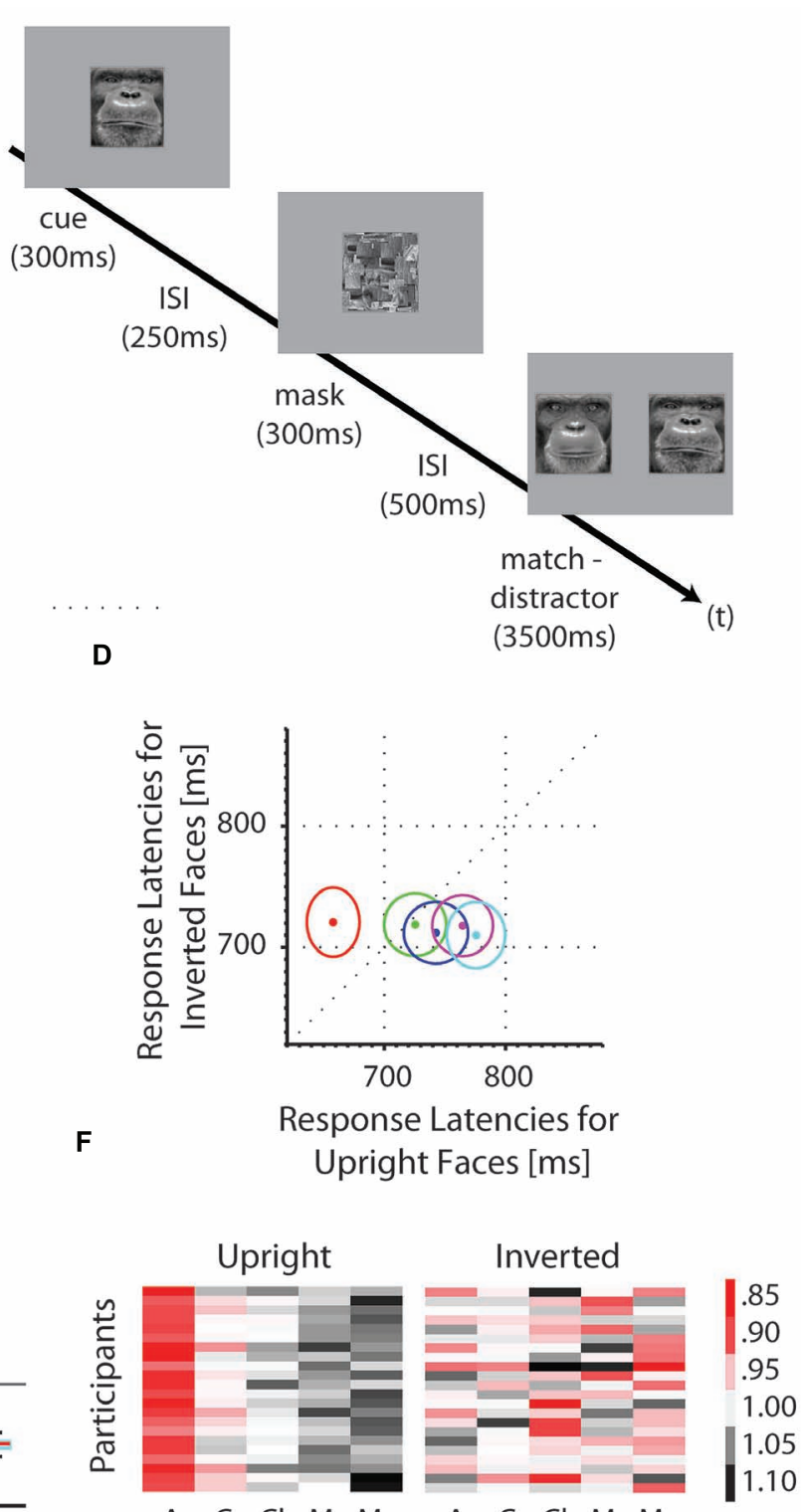

A C Ch MqMm A C Ch MqMm

for inverted faces in lighter colors. (D) Absolute reaction times of upright versus inverted faces. Mean reaction times for upright and inverted faces are shown as dots. Colors correspond to the colors in (C). Circles indicate the standard errors. (E) Differences of normalized reaction times of upright and inverted faces. Reaction times were normalized for each face class by the mean of the corresponding class. Scores for upright faces were subtracted from those of inverted faces for each face class. Positive values indicate an advantage for upright above inverted faces in terms of discrimination speed, while negative values indicate an advantage for inverted above upright faces. (F) Normalized reaction times for each participant. The color code represents the relative reaction times, with red being fast, black being slow and white intermediate. Values above zero indicate slower responses than the mean response $(=0)$; values below zero indicate faster responses than the mean response. 
A

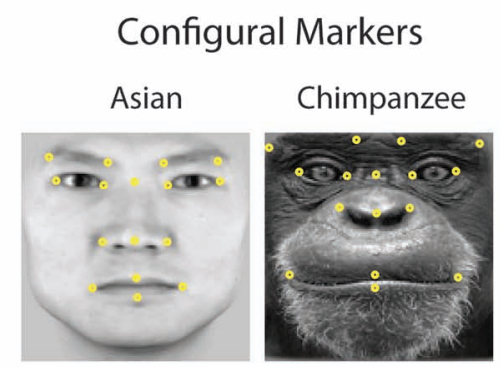

c Multi-dimensional scaling (MDS)

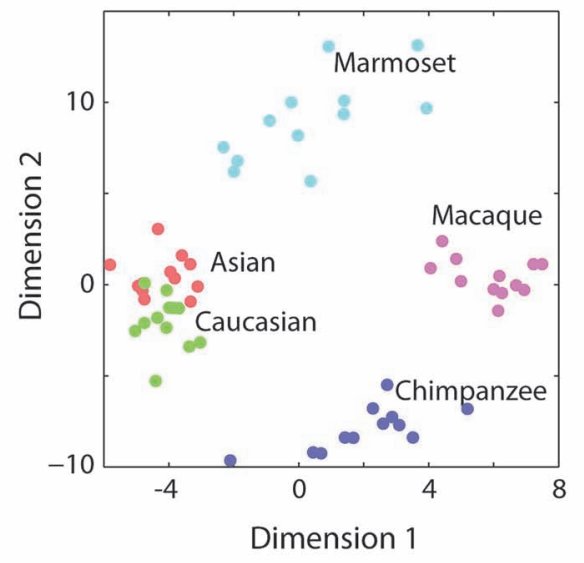

B

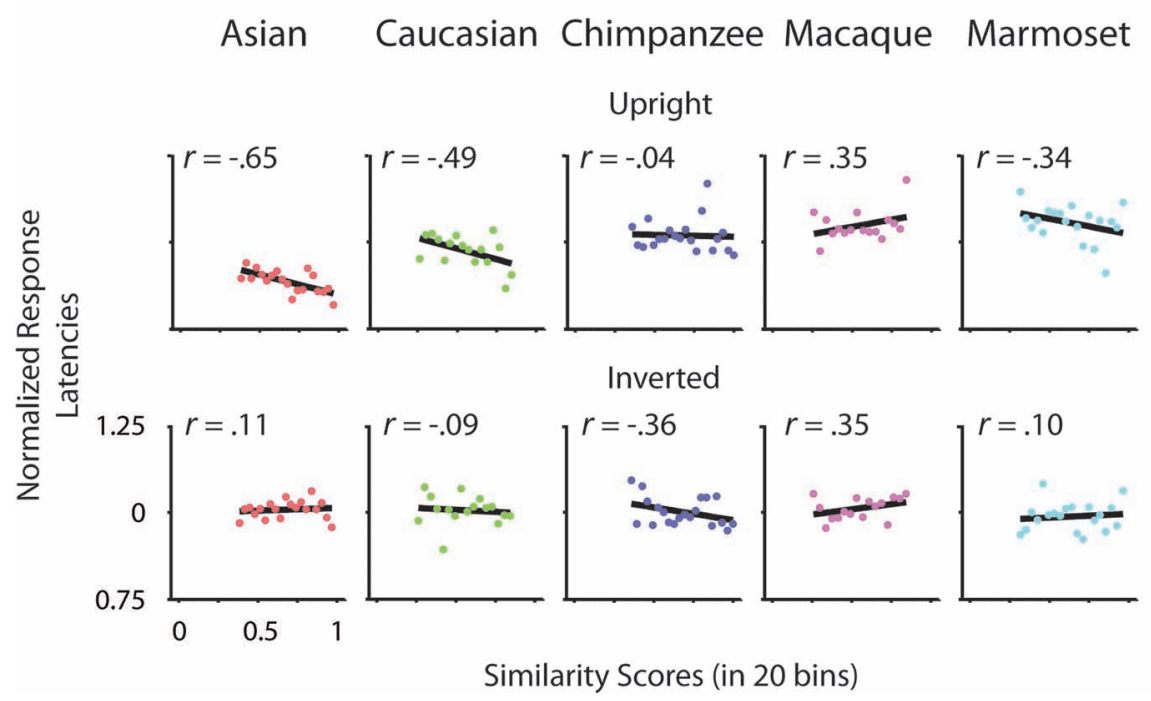

FIGURE 2 | Configural analysis. (A) Configural markers. Illustrative arrangement of facial markers on Asian and chimpanzee faces (yellow dots). Corresponding points as shown were selected for each individual face. (B) Differences of normalized reaction times of upright and inverted faces as a function of configural similarity. Differences of normalized reaction times of upright and inverted faces were correlated with the binned values of similarity scores drawn from the configural arrangement of pairs of faces. Black lines indicate the correlation. (C) Multidimensional scaling (MDS) space of configural similarities among faces and face classes. $p<0.05$; chimpanzee $r=-0.54, p<0.01$; macaque $r=-0.59$, $p<0.01$; marmoset $r=-0.47, p=0.05)$. The similarity scores of the nose regions were not correlated with reaction times of upright faces in all face classes, but for the macaque and marmoset monkeys (Asian: $r=0.08, p=0.62$; Caucasian: $r=-0.11$, $p=0.33$; chimpanzee $r=-0.16, p=0.25$; macaque $r=-0.41$, $p<0.05$; marmoset $r=-0.52, p=0.01)$. The similarity scores of the mouth region were negatively correlated with the reaction times of upright face in Caucasian and chimpanzee faces (Asian: $r=0.29, p=0.88$; Caucasian: $r=-0.64, p<0.001$; chimpanzee $r=-0.52, p<0.05$; macaque $r=0.29, p=0.89$; marmoset $r=0.18, p=0.76)$. In contrast, the similarity scores of all parts were negatively correlated with the reaction times of inverted faces with some exceptions: the chimpanzee's nose and the macaque's and marmoset's mouths did not elicit any correlation (Eyes: Asian: $r=-0.65, p<0.001$; Caucasian: $r=-0.43, p<0.05$; chimpanzee $r=-0.65, p<0.01$; macaque $r=-0.60, p<0.01$; marmoset $r=-0.74, p=0.001$; Nose: Asian: $r=-0.54, p<0.05$; Caucasian: $r=-0.48, p<0.05$; chimpanzee $r=-0.06, p=0.40$; macaque $r=-0.48, p<0.05$; marmoset $r=-0.59, p<0.01$; Mouth: Asian: $r=-0.61, p<0.01$; Caucasian: $r=-0.65, p<0.01$; chimpanzee $r=-0.57, p<0.01$; macaque $r=0.56, p=0.99$; marmoset $r=0.40, p=0.95)$.

We found a relative increase of reaction times for the discrimination of OREs as well as OSEs. The OSE for upright faces becomes more pronounced with increasing distance from the human species on the evolutionary timeline. We further showed that while face inversion has a marginal or facilitative effect on faces of the other race, it has a drastic deteriorative effect on faces of the same race (see discussion). Same-race faces showed a strong 


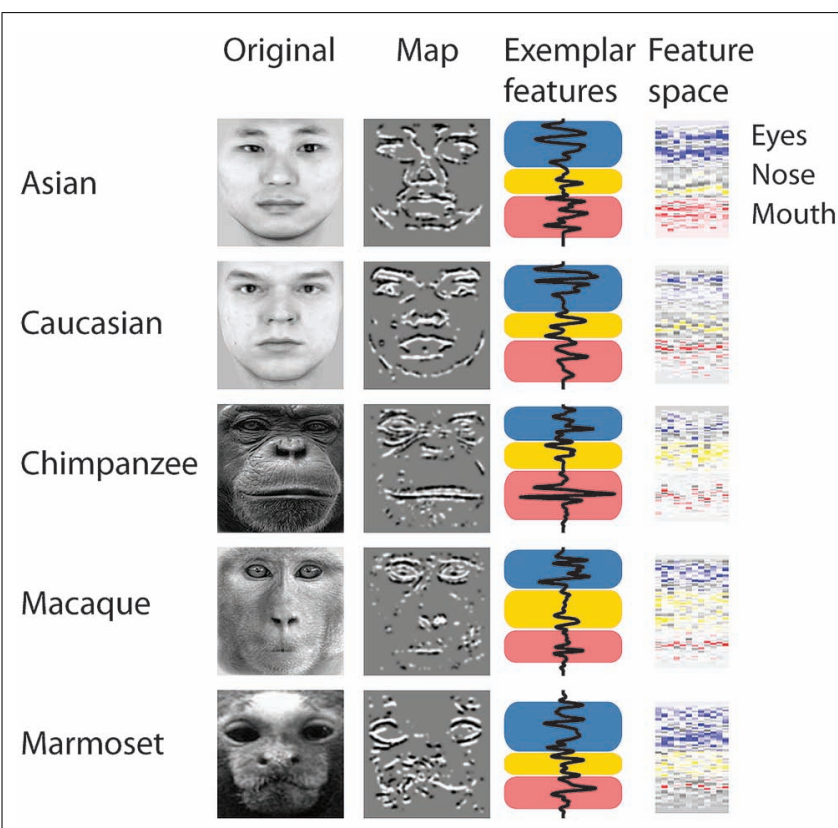

FIGURE 3 | Face part extraction, part-based analysis. The process of extraction face parts from an original image (non-normalized images are shown here for illustration). Black and white colors in the "Map" plots illustrate the ravines of the face. Colors in the "Exemplar features" and "Feature space" plots illustrate the face region (blue = eyes;

yellow = nose; red = mouth). The "Feature space" panels show the feature profiles ( $y$-axis) for each stimulus ( $x$-axis).

negative correlation between reaction times and configural similarity, indicating configural sensitivity to a great extent. OREs showed a relatively weaker negative correlation between reaction times and configural similarity, indicating weaker configural sensitivity. All other faces showed negative correlations between reaction times and similarity scores of certain facial parts, suggesting processing mechanisms with focus on individual - likely the most distinctive - face parts.

\section{DISCUSSION}

We addressed how configural processing and similarity at subordinate-level (within-class) affect discrimination performance for faces. Only a few accounts, to date, have taken similarity among stimulus samples into consideration when evaluating discrimination performances (Martin-Malivel and Okada, 2007; Dahl et al., 2013a). Here we evaluate the extent to which participants rely on configural similarity among the two samples of a stimulus pair. A related study showed that with increasing similarity among stimuli configural processing increased (Hsiao and Cheung, 2011). We determined the reliance on configural processing by looking at upright as opposed to inverted faces. The results indicate that participants relied on configural information more strongly with increasing configural similarity between the two faces presented. This was true only for the own face class, to a lesser degree for the ORE class and only in upright presentation condition. In other words, the more similar the configurations of two faces was the more the system relied on configural processing in order to successfully discriminate the two faces.
We also addressed the extent to which the participants relied on specific facial parts. We expect faces of other species and races as well as inverted own-race and own-species faces to be processed in a stronger part-based manner than upright ownspecies and own-race faces. The part-based analysis revealed a general relationship between similarity of parts and discrimination performance for all face classes. In more detail, the eyes played a crucial role for all types of classes; the mouth played a more important role for Caucasian and chimpanzee faces, not so much for monkey faces; while in contrast the nose played a more critical role in monkey faces as opposed to human and chimpanzee faces. As can be seen in Figure 1A, left column, the chimpanzee faces, in contrast to the monkey faces, contain a large variance in the mouth area. In other words, differential diagnostic features are used for the OSE classes. In the upright own-face class, only the similarity of eyes was negatively correlated with the performances, which might be caused by the strong eye dominance in face recognition, as described in humans (Barton et al., 2006) and monkeys (Keating and Keating, 1993; Gothard et al., 2004; Dahl et al., 2007). This, however, drastically changes when the own-class faces were presented inverted: similarity of all parts were correlated negatively with the performance scores, speaking for a rather distributed account on discriminating inverted own-race/species faces. Together, these findings consistently reflect the involvement of two processing strategies, configural and part-based processing, depending on the type of face and the presentation condition. Along the lines, eye tracking studies in humans (Barton et al., 2006) and monkeys (Dahl et al., 2009) showed that eye gaze distribution were more compact on the eye region of upright conspecific faces and more distributed across facial parts for upright non-conspecific and inverted faces. Here, we found stronger focus on the eyes only in the upright own-race/species faces and more distributed reliance on several facial parts for upright other-race/species faces as well as inverted faces. In combination with the stronger reliance on configural similarity for upright own-race/species faces, we can disentangle the two processing mechanisms.

How can we interpret our findings? A critical component in face perception is the level of expertise the observer has with the face class presented. Expertise of the observer was found to influence ORE. Configural information is used to a greater extent for own-race as opposed to OREs (Tanaka et al., 2004; Michel et al., 2006): Caucasians showed a larger whole-face advantage (in comparison to using individual facial parts) for own-race as opposed to OREs, while Asians, living in a society predominantly populated by Caucasians, showed an equal whole-face advantage for both types of faces. Similarly, expertise influences the OSE: humans and monkeys showed higher sensitivity toward so-called secondorder relational properties (Adachi et al., 2009; Dahl et al., 2010, 2011), i.e., the spatial dimensions among facial parts (Tanaka and Farah, 1991), and diagnostic scanning patterns indicating configural processing of own-species faces and part-based processing of OSEs (Dahl et al., 2009). Accordingly, adult humans more easily detect slight spatial changes in human faces relative to monkey faces, while the performance is impaired for human faces when presented in inverted condition (Mondloch et al., 2006). Further, captive chimpanzees were tested on discriminating chimpanzee 
A
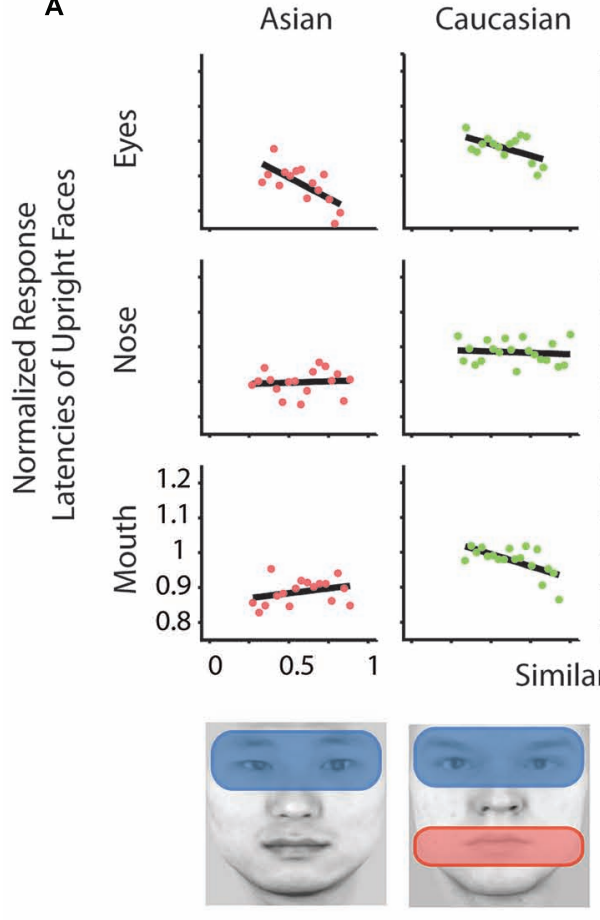

B
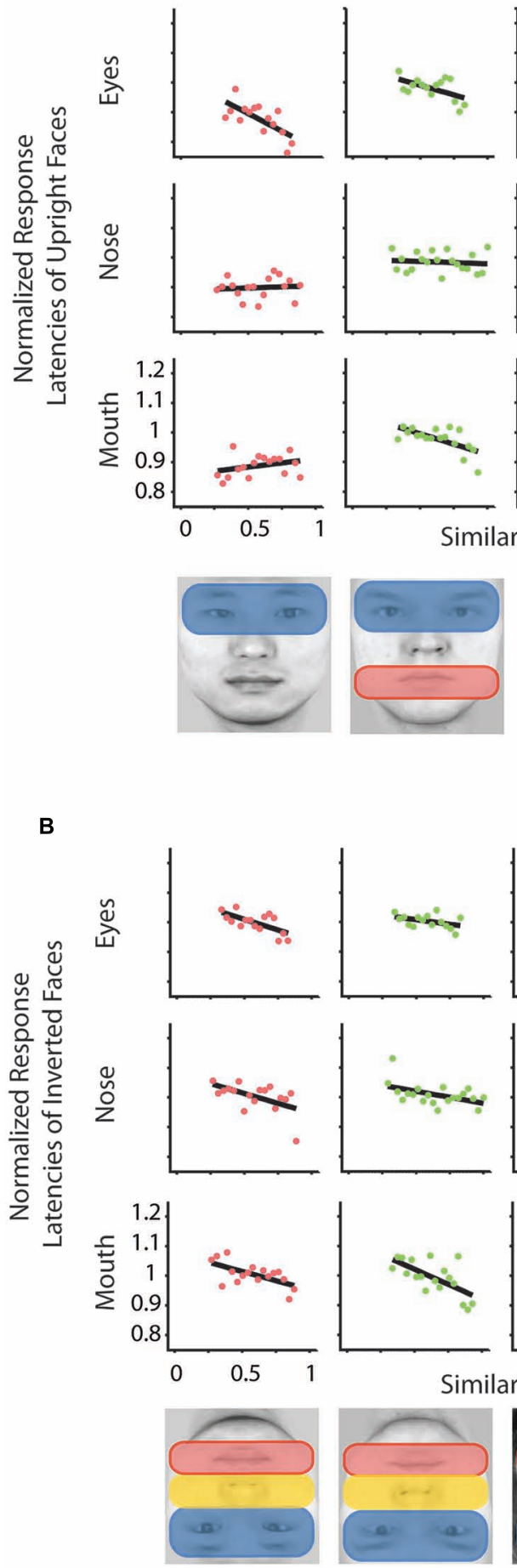

\section{Upright}

Caucasian Chimpanzee Macaque Marmoset
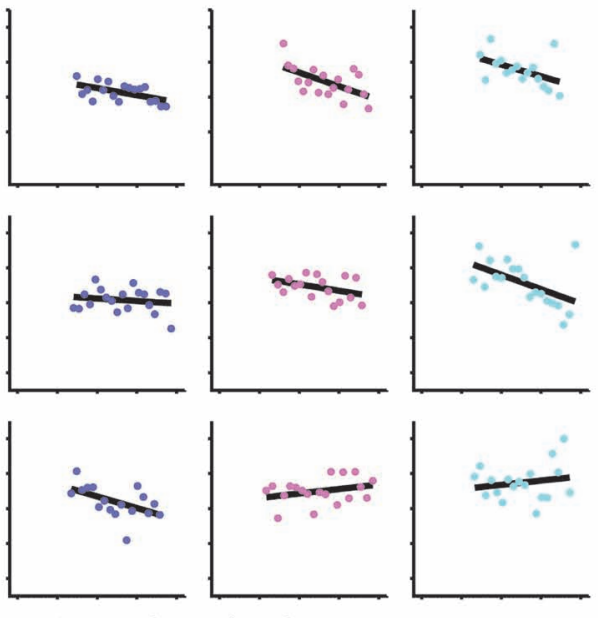

Similarity Scores (in 20 bins)
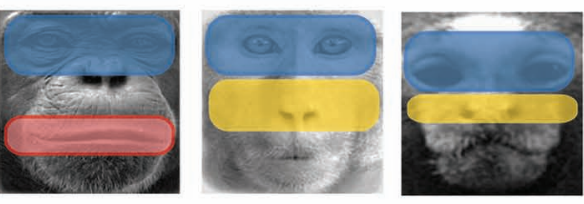

Inverted
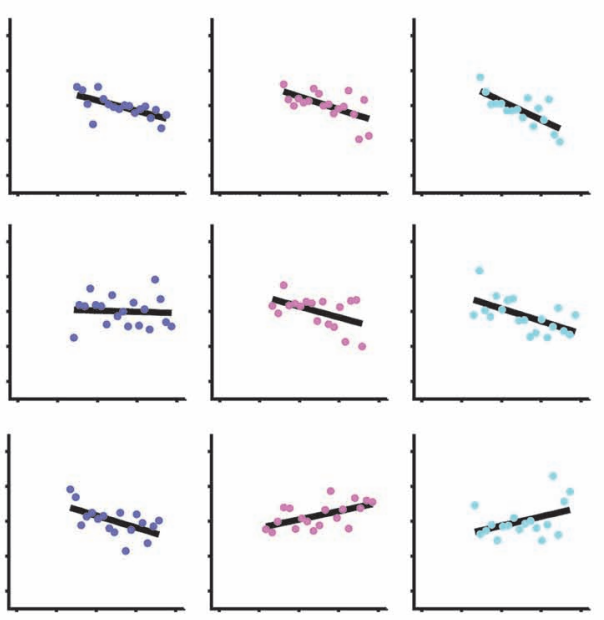

Similarity Scores (in 20 bins)
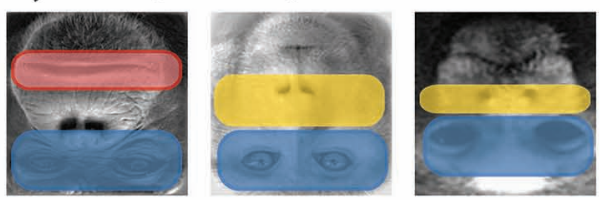

Negative Correlation

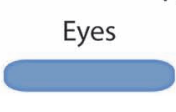

FIGURE 4 | Normalized reaction times of upright and inverted faces as a function of similarity scores drawn from face parts. Normalized reaction the binned values of similarity scores drawn from the face parts of pairs of faces. Black lines indicate the correlation. Below the graphs the significantly negatively correlated areas are highlighted. 
and human faces (Dahl et al., 2013a). Young chimpanzees (around 10 years of age) showed a clear advantage for chimpanzee faces above human faces; however, the advantage turned into a disadvantage with increasing exposure to human faces and limited exposure to chimpanzee faces: older chimpanzees (around 30 years of age) showed an advantage for human above chimpanzee faces. In other words the sensitivity toward one class of faces adapted toward another class of faces more strongly exposed to over decades. These same chimpanzees showed a more pronounced face inversion effect (Dahl et al., 2013b) and a more pronounce left-chimeric face bias (indicating dominant right-hemispheric processing; Dahl etal., 2013c) for faces of the expert category. Monkey experts turn out to be more accurate than non-experts at identifying faces of expertise and were more affected by inversion of those monkey faces (Dufour and Petit, 2010).

As shown in this study, the similarity of two faces influences how fast we can differentiate the two. In terms of the own-race face configuration, the more closer the configural arrangements of two faces are, the more the observer relied on a configural approach. While we report an own-race advantage in terms of reaction times, i.e., faster discrimination for own-race than OREs, we find that configural information plays a role in both ownrace as well as OREs. However, it needs to be clearly stated that the effect size is greater for own-race than OREs, with the latter being significant at exactly $5 \%$. In other words, a face class which is morphologically close to the own-face class can be treated to some extent configurally [see Figure $\mathbf{2 C}$ for morphological similarity in a multidimensional scaling (MDS) space]. However, with increasing morphological distance OSEs, configural information cannot be successfully used for face discrimination. In accordance with this interpretation, own-race faces have been reported to be processed more configurally than OREs (Michel et al., 2006). Further, according to the expertise (contact) hypothesis (Bukach et al., 2006), the extent to which OREs were accessed configurally, might well reflect the level of exposure of our participants to Caucasian faces. Critically, our results suggest that there is no onoff state between part-based and configural processing strategies, but rather a continuous change of processing strategies along the grades of similarity with own-class faces.

How can we explain the trend toward facilitation of primate faces when inverted as opposed to upright? First, it has to be clearly stated that there was no significant inversion effect for face classes of the closer evolutionary relatives to humans, chimpanzees and macaques. However, marmoset monkey faces showed a difference, and chimpanzee and macaque faces showed a trend toward a face inversion effect. A possible explanation is as follows: when the non-human primate faces were presented upright, the visual system attempts to process these faces like human (here Asian) faces due to a default approach to facelike stimuli. However, given the high degree of morphological difference between non-human primate and human faces, defaulttemplate processing fails for non-human primates to a great extent. This would explain an increasing deterioration with increasing evolutionary distance. Once the faces were presented inverted, they were all treated equally and processed according to a partbased manner, hence resulting in relatively equal reaction times for discriminating human and non-human primate faces. In a recent computational model (SCORE; Dahl et al., accepted), we found that, indeed, the representational structure of Asian faces was more different in its higher dimensional components to the representational structure of chimpanzee faces than Caucasian faces. This supports the idea that applying a human face template onto a non-human face reduces the discrimination power drastically.

Face perception is governed by two processing mechanisms, configurally, and part-based processing, and presumably a mixture of both. This study shows that own-class faces tend to follow configural processing rules, while other face classes are governed more strongly by part-based processing. The interplay of configural and part-based processing is influenced by a number of factors: (1) Expertise in discrimination faces drives the perceptual system toward a configural strategy. (2) Morphological similarity among face classes determines the extent to which the perceptual system is able to generalize from the expert face class to a non-expert face class. In our study, Asian-face experts can to some degree make use of configural information in Caucasian faces due to the close morphological distance of Asian and Caucasian faces. (3) Inversion disrupts access to configural information largely and breaks down the processing strategies of all face classes to an analytic part-based manner. Hence, these factors influence the general discrimination ability of various face classes and manifest in effects like the ORE and OSE.

\section{AUTHOR CONTRIBUTIONS}

Christoph D. Dahl conceived, designed and performed the experiments; Christoph D. Dahl analyzed the data; Christoph D. Dahl, Malte J. Rasch and Chien-Chung Chen contributed reagents/materials/analysis tools; Christoph D. Dahl wrote the paper. All authors discussed the results and commented on the manuscript.

\section{ACKNOWLEDGMENTS}

This research was financially supported by the AIM for Top University Excellent Research Project The visual analysis of expression representation -103R892101 (Chien-Chung Chen) and partly by NSFC Grant 31371109 (Malte J. Rasch). We thank Christian Wallraven for useful comments, Heinrich Bulthoff and the ScanLab of the Max Planck Institute for Biological Cybernetics for contributing the Asian and Caucasian face pictures, Nikos Logothetis for contributing macaque and marmoset face pictures and the Great Ape Research Institute (GARI) and the Primate Research Institute of Kyoto University for the chimpanzee face pictures.

\section{APPROVING COMMITTEE}

The study was approved by the Research Ethic Committee of National Taiwan University and followed the principles of Helsinki Declaration and local laws and regulations.

\section{REFERENCES}

Adachi, I., Chou, D. P., and Hampton, R. R. (2009). Thatcher effect in monkeys demonstrates conservation of face perception across primates. Curr. Biol. 19, 1270-1273. doi: 10.1016/j.cub.2009.05.067

Barton, J. J., Radcliffe, N., Cherkasova, M. V., Edelman, J., and Intriligator, J. M. (2006). Information processing during face recognition: the effects of familiarity, inversion, and morphing on scanning fixations. Perception 35, 1089-1105. doi: $10.1068 / \mathrm{p} 5547$ 
Bothwell, R. K., Brigham, J. C., and Malpass, R. S. (1989). Cross-Racial Identification. Pers. Soc. Psychol. Bull. 15, 19-25. doi: 10.1177/0146167289151002 Brainard, D. H. (1997). The psychophysics toolbox. Spat. Vis. 10, 433-436. doi: $10.1163 / 156856897 X 00357$

Bruce, V., and Young, A. (1986). Understanding face recognition. Br. J. Psychol. 77, 305-327. doi: 10.1111/j.2044-8295.1986.tb02199.x

Bukach, C. M., Gauthier, I., and Tarr, M. J. (2006). Beyond faces and modularity: the power of an expertise framework. Trends Cogn. Sci. 10, 159-166. doi: 10.1016/j.tics.2006.02.004

Bulthoff, I. (2009). Sex categorization is influenced by facial information about identity. Perception 38, 78-78.

Dahl, C. D., and Adachi, I. (2013). Conceptual metaphorical mapping in chimpanzees (Pan troglodytes). elife 2:e00932. doi: 10.7554/eLife.00932

Dahl, C. D., Logothetis, N. K., Bulthoff, H. H., and Wallraven, C. (2010). The Thatcher illusion in humans and monkeys. Proc. Biol. Sci. 277, 2973-2981. doi: 10.1098/rspb.2010.0438

Dahl, C. D., Logothetis, N. K., Bulthoff, H. H., and Wallraven, C. (2011). Secondorder relational manipulations affect both humans and monkeys. PLoS ONE 6:e25793. doi: 10.1371/journal.pone.0025793

Dahl, C. D., Logothetis, N. K., and Hoffman, K. L. (2007). Individuation and holistic processing of faces in rhesus monkeys. Proc. Biol. Sci. 274, 2069-2076. doi: 10.1098/rspb.2007.0477

Dahl, C. D., Rasch, M. J., Tomonaga, M., and Adachi, I. (2013a). Developmental processes in face perception. Nat. Sci. Rep. 3, 1044. doi: 10.1038/srep01044

Dahl, C. D., Rasch, M. J., Tomonaga, M., and Adachi, I. (2013b). The face inversion effect in non-human primates revisited - an investigation in chimpanzees (Pan troglodytes). Sci. Rep. 3, 2504. doi: 10.1038/srep02504

Dahl, C. D., Rasch, M. J., Tomonaga, M., and Adachi, I. (2013c). Laterality Effect for Faces in Chimpanzees (Pan troglodytes). J. Neurosci. 33, 13344-13349. doi: 10.1523/JNEUROSCI.0590-13.2013

Dahl, C. D., Wallraven, C., Bulthoff, H. H., and Logothetis, N. K. (2009). Humans and macaques employ similar face-processing strategies. Curr. Biol. 19, 509-513. doi: 10.1016/j.cub.2009.01.061

de Haan, M., Pascalis, O., and Johnson, M. H. (2002). Specialization of neural mechanisms underlying face recognition in human infants. J. Cogn. Neurosci. 14 199-209. doi: 10.1162/089892902317236849

Dufour, V., Coleman, M., Campbell, R., Petit, O., and Pascalis, O. (2004). On the species-specificity of face recognition in human adults. Curr. Psychol. Cogn. 22, 315-333.

Dufour, V., Pascalis, O., and Petit, O. (2006). Face processing limitation to own species in primates: a comparative study in brown capuchins, Tonkean macaques and humans. Behav. Processes 73, 107-113. doi: 10.1016/j.beproc.2006.04.006

Dufour, V., and Petit, O. (2010). Recognition of monkey faces by monkey experts. J. Ethol. 28, 231-238. doi: 10.1007/s10164-009-0174-8

Ekman, P., Friesen, W. V., Osullivan, M., and Scherer, K. (1980). Relative importance of face, body, and speech in judgments of personality and affect. J. Pers. Soc Psychol. 38, 270-277. doi: 10.1037/0022-3514.38.2.270

Freire, A., Lee, K., and Symons, L. A. (2000). The face-inversion effect as a deficit in the encoding of configural information: direct evidence. Perception 29, 159-170. doi: $10.1068 / \mathrm{p} 3012$

Gothard, K. M., Erickson, C. A., and Amaral, D. G. (2004). How do rhesus monkeys (Macaca mulatta) scan faces in a visual paired comparison task? Anim. Cogn. 7, 25-36. doi: 10.1007/s10071-003-0179-6

Gunduz, A., and Krim, H. (2003). "Facial feature extraction using topological methods," in Proceedings of IEEE 2003 International Conference on Image Processing, Vol. 1, (Barcelona: IEEE), 673-676. doi: 10.1109/ICIP.2003.1247051

Hsiao, J. H., and Cheung, K. C. F. (2011). "Computational exploration of the relationship between holistic processing and right hemisphere lateralization in featural and configural recognition tasks," in Proceedings of the 33rd Annual Conference of the Cognitive Science Society, eds C. H. L. Carlson and T. F. Shipley (Austin, TX: Cognitive Science Society), 2592-2597.

Hugenberg, K., Young, S. G., Bernstein, M. J., and Sacco, D. F. (2010). The categorization-individuation model: an integrative account of the other-race recognition deficit. Psychol. Rev. 117, 1168-1187. doi: 10.1037/a0020463

Keating, C. F., and Keating, E. G. (1993). Monkeys and mug shots: cues used by rhesus monkeys (Macaca mulatta) to recognize a human face. J. Comp. Psychol. 107, 131-139. doi: 10.1037/0735-7036.107.2.131
Kwon, Y. H., and da Vitoria Lobo, N. (1999). Age classification from facial images. Comput. Vis. Image Underst. 74, 1-21. doi: 10.1006/cviu.1997.0549

Li, C. M., Li, Y. S., Zhuang, Q. D., and Xiao, Z. Z. (2004). “The face localization and regional features extraction," in Proceedings of IEEE 2004 International Conference on Machine Learning and Cybernetics, Shanghai, Vol. 6, 3835-3840. doi: 10.1109/ICMLC.2004.1380504

Lindsay, D. S., Jack, P. C., and Christian, M. A. (1991). Other-race face perception. J. Appl. Psychol. 76, 587-589. doi: 10.1037/0021-9010.76.4.587

Marr, D., and Nishihara, H. K. (1978). Representation and recognition of the spatial organization of three-dimensional shapes. Proc. R. Soc. Lond. B Biol. Sci. 200, 269-294. doi: 10.1098/rspb.1978.0020

Martin-Malivel, J., and Okada, K. (2007). Human and chimpanzee face recognition in chimpanzees (Pan troglodytes): role of exposure and impact on categorical perception. Behav. Neurosci. 121, 1145-1155. doi: 10.1037/0735-7044. 121.6.1145

Meissner, C. A., and Brigham, J. C. (2001). Thirty years of investigating the own-race bias in memory for faces - A meta-analytic review. Psychol. Public Policy Law 7, 3-35. doi: 10.1037/1076-8971.7.1.3

Meissner, C. A., Brigham, J. C., and Butz, D. A. (2005). Memory for own- and other-race faces: a dual-process approach. Appl. Cogn. Psychol. 19, 545-567. doi: 10.1002/acp.1097

Michel, C., Caldara, R., and Rossion, B. (2006). Same-race faces are perceived more holistically than other-race faces. Vis. Cogn. 14, 55-73. doi: $10.1080 / 13506280500158761$

Michel, C., Corneille, O., and Rossion, B. (2007). Race categorization modulates holistic face encoding. Cogn. Sci. 31, 911-924. doi: 10.1080/03640210701530805 Mondloch, C. J., Maurer, D., and Ahola, S. (2006). Becoming a face expert. Psychol. Sci. 17, 930-934. doi: 10.1111/j.1467-9280.2006.01806.x

Pascalis, O., and Bachevalier, J. (1998). Face recognition in primates: a cross-species study. Behav. Processes 43, 87-96. doi: 10.1016/S0376-6357(97)00090-9

Pascalis, O., De Haan, M., and Nelson, C. A. (2002). Is face processing speciesspecific during the first year of life? Science 296, 1321-1323. doi: 10.1126/science. 1070223

Pelli, D. G. (1997). The VideoToolbox software for visual psychophysics: transforming numbers into movies. Spat. Vis. 10, 437-442. doi: 10.1163/156856897X00366

Scott, L. S., and Fava, E. (2013). The own-species face bias: a review of developmental and comparative data. Vis. Cogn. 21, 1364-1391. doi: $10.1080 / 13506285.2013 .821431$

Scott, L. S., Shannon, R. W., and Nelson, C. A. (2005). Behavioral and electrophysiological evidence of species-specific face processing. Cogn. Affect. Behav. Neurosci. 5, 405-416. doi: 10.3758/CABN.5.4.405

Tanaka, J. W., and Farah, M. J. (1991). 2nd-Order relational properties and the inversion effect - testing a theory of face perception. Percept. Psychophys. 50, 367-372. doi: 10.3758/BF03212229

Tanaka, J. W., Kiefer, M., and Bukach, C. M. (2004). A holistic account of the ownrace effect in face recognition: evidence from a cross-cultural study. Cognition 93, B1-B9. doi: 10.1016/j.cognition.2003.09.011

Tanaka, J. W., and Pierce, L. J. (2009). The neural plasticity of other-race face recognition. Cogn. Affect. Behav. Neurosci. 9, 122-131. doi: 10.3758/CABN.9.1.122

Conflict of Interest Statement: The authors certify that they have no affiliations with or involvement in any organization or entity with any financial interest, or non-financial interest in the subject matter or materials discussed in this manuscript.

Received: 07 April 2014; accepted: 05 September 2014; published online: 19 September 2014.

Citation: Dahl CD, Rasch MJ and Chen C-C (2014) The other-race and other-species effects in face perception - a subordinate-level analysis. Front. Psychol. 5:1068. doi: $10.3389 /$ fpsyg.2014.01068

This article was submitted to Cognitive Science, a section of the journal Frontiers in Psychology.

Copyright (C) 2014 Dahl, Rasch and Chen. This is an open-access article distributed under the terms of the Creative Commons Attribution License (CC BY). The use, distribution or reproduction in other forums is permitted, provided the original author(s) or licensor are credited and that the original publication in this journal is cited, in accordance with accepted academic practice. No use, distribution or reproduction is permitted which does not comply with these terms. 